\title{
Fusing Complex Big Data Sets to Understand Consumer's Online Relationships that Create In-Store Retail Bonding: An Abstract
}

\author{
Don Schultz and Martin P. Block
}

\begin{abstract}
In this exploratory study, multiple consumer online and offline actions, along with external factors were analyzed and then fused with existing and gathered in-store consumer behavioral data sets to develop a richer and more complete view of the various interactions which lead to consumer bonding to a retail chain. While the time period analyzed was limited, the richness and detail of the data enabled a more complete view of how online consumer activities interact with and explain in-store consumer behaviors and the sales results which occur. In addition, two other external variables, weather and adjacency of competitive retail facilities, were used to enhance the "real world" situation in which consumers operate. These provide additional insights into why consumers interact with retailers the way they do. This analysis provides guidelines and insights into the potential value of more detailed and complex retail store/consumer analyses going forward. Based on the results of this initial study, it appears the approaches used in this analysis are transferrable to other situations and might be used around the globe.

Of great importance is evidence that a grounded theory approach (Ellis 1993) might be the optimal one in analyzing consumer behavior at the retail level. The authors argue that it is the combining of multiple inputs that enables the exploration of big data using a "test, evaluate, and measure" approach, which provides the greatest insights into how consumers actually behave in the marketplace. They further argue that this approach likely surpasses traditional limited hypothesis testing now used in retail analytics.
\end{abstract}

References Available Upon Request

D. Schultz $\cdot$ M. P. Block $(\bowtie)$

Northwestern University, Evanston, IL, USA

e-mail: dschultz@northwestern.edu; mp-block@northwestern.edu

C) Academy of Marketing Science 2019 